\title{
Nitrogen X-ray absorption in the local ISM
}

\author{
Efrain Gatuzz, ${ }^{1 \star}$ Javier A. García ${ }^{2,3}$ and Timothy R. Kallman ${ }^{4}$ \\ ${ }^{1}$ Max-Planck-Institut für extraterrestrische Physik, Gießenbachstraße 1, D-85748 Garching, Germany \\ ${ }^{2}$ Cahill Center for Astronomy and Astrophysics, California Institute of Technology, Pasadena, CA 91125, USA \\ ${ }^{3}$ Dr. Karl Remeis-Observatory and Erlangen Centre for Astroparticle Physics, Sternwartstr 7, D-96049 Bamberg, Germany \\ ${ }^{4}$ NASA Goddard Space Flight Center, Greenbelt, MD 20771, USA
}

Accepted 2021 April 21. Received 2021 April 21; in original form 2020 September 17

\begin{abstract}
Nitrogen is one of the most abundant metals in the interstellar medium (ISM), and thus it constitutes an excellent test to study a variety of astrophysical environments, ranging from nova to active galactic nuclei. We present a detailed analysis of the gaseous component of the N K-edge using high-resolution XMM-Newton spectra of 12 Galactic and 40 extragalactic sources. For each source, we have estimated column densities for N I, N II, N III, N V, N VI, and N VII ionic species, which trace the cold, warm, and hot phases of the local Galactic ISM. We have found that the cold-warm component column densities decrease with the Galactic latitude, while the hot component does not. Moreover, the cold column density distribution is in good agreement with UV measurements. This is the first detailed analysis of the nitrogen K-edge absorption due to ISM using high-resolution X-ray spectra.
\end{abstract}

Key words: ISM: abundances - ISM: atoms - ISM: structure-Galaxy: structure-X-rays: ISM.

\section{INTRODUCTION}

The interstellar medium (ISM), defined as gas and dust between stars, is a key ingredient in the Galactic dynamics. The ISM shows multiple phases, characterized by different gas temperatures which vary from 10 to $10^{6} \mathrm{~K}$ (e.g. McKee \& Ostriker 1977; Falgarone et al. 2005; Draine 2011; Jenkins \& Tripp 2011; Stanimirović \& Zweibel 2018). In this sense, high-resolution X-ray spectroscopy is a powerful technique to study such environment because, due to their high energy, X-ray photons interact with the cold (including molecules and dust), warm, and hot components (Juett, Schulz \& Chakrabarty 2004; Juett et al. 2006; Pinto et al. 2010; Liao, Zhang \& Yao 2013; Pinto et al. 2013; Luo \& Fang 2014; Nicastro et al. 2016a; Gupta, Mathur \& Krongold 2017; Gatuzz \& Churazov 2018; Gatuzz et al. 2018b).

Among the most abundant metals in the ISM, nitrogen constitutes an excellent diagnostic tool to study a variety of astrophysical environments. For example, Ness et al. (2003) identified H- and He-like $\mathrm{N}$ absorption lines by a white dwarf outflow following the outburst of nova V4743 Sagittarii. Steenbrugge et al. (2005a) identified an $\mathrm{N}$ VI Ly $\alpha$ absorption line associated with a warm absorber in the XMM-Newton X-ray spectra of the Seyfert 1 galaxy IC 4329A. Steenbrugge et al. (2005b) identified an N V K $\alpha$ absorption line as $29.42 \AA$ in the outflow of the Seyfert 1 galaxy NGC 5548. Smith, Page \& Branduardi-Raymont (2007) performed a detailed analysis of the soft X-ray spectrum of the Seyfert 1 galaxy Mrk 509 using XMMNewton observations. They found three warm absorber phases traced, among others, by N VI and N VII absorption lines. Ramírez et al.

^E-mail: efraingatuzz@gmail.com
(2008) identified N VI K $\alpha$ and N VI K $\beta$ absorption lines in the warm absorber of the MR 2251-178 quasar, tracing an outflow of ionized material. Ness et al. (2011) analysed XMM-Newton observations of the fast classical nova V2491 Cyg. They found absorption lines due to $\mathrm{N}$ I associated with the ISM and photospheric lines ionic species such as N VI and N VII, tracing the dynamics of the ejecta.

In order to model the complexity of the photoabsorption K-edge, located at the 24-32 $\AA$ wavelength band, the accuracy of atomic data is crucial, to avoid misidentification and misinterpretation of the observed absorption features. In the last few years we have performed benchmarking of the atomic data by comparing theoretical calculations, astronomical observations, and laboratory measurements for species found in the ISM such as carbon (Gatuzz et al. 2018a), oxygen (García et al. 2005; Gorczyca et al. 2013; Gatuzz et al. 2013a, b), neon (Gatuzz et al. 2015), magnesium (Hasoğlu et al. 2014), and silicon (Gatuzz et al. 2020). Following such studies, we present an analysis of the N K-edge absorption region using XMM-Newton observations of low-mass X-ray binaries (LMXBs) and extragalactic sources. We describe the data sample and the spectral fitting procedure in Section 2. Section 3 shows a discussion of the results obtained from the fits. Finally, Section 4 summarizes the main results of our analysis.

\section{X-RAY OBSERVATIONS AND SPECTRAL FITTING}

We have compiled a data sample of 12 LMXBs and 40 extragalactic sources from the XMM-Newton Science Archive (XSA). We selected observations with more than 1000 counts in the 24-32 $\AA$ band (i.e. the $\mathrm{N}$ photoionization $\mathrm{K}$-edge absorption region). In order to get an unbiased sample, we did not impose any constraints in the 
Table 1. List of Galactic observations analysed.

\begin{tabular}{lccccc}
\hline Source & $\begin{array}{c}\text { Galactic } \\
\text { coordinates }\end{array}$ & $\begin{array}{c}\text { Distance } \\
(\mathrm{kpc})\end{array}$ & $N(\mathrm{HI})$ & $\begin{array}{c}\text { Exposure } \\
\text { time }(\mathrm{ks})\end{array}$ & $\begin{array}{c}\text { Number counts } \\
(24-32 \AA)\end{array}$ \\
\hline 4U 1254-69 & $(303.4,-6.4)$ & $13.0 \pm 3.0^{a}$ & 3.46 & 233 & 66936 \\
4U 1543-62 & $(321.76,-6.34)$ & $7.0^{b}$ & 3.79 & 50 & 35103 \\
4U 1636-53 & $(332.9,-4.8)$ & $6.0 \pm 0.5^{c}$ & 4.04 & 379 & 137151 \\
4U 1735-44 & $(346.0,-6.9)$ & $9.4 \pm 1.4^{d}$ & 3.96 & 107 & 85494 \\
4U 1820-30 & $(2.7,-7.9)$ & $7.6 \pm 0.4^{e}$ & 1.33 & 81 & 381038 \\
4U 1957+11 & $(51.31,-9.33)$ & $20<d<40^{f}$ & 1.98 & 85 & 65478 \\
Aq1 X-1 & $(37.7,-4.1)$ & $5.2 \pm 0.8^{d}$ & 4.30 & 165 & 43229 \\
Cygnus X-2 & $(87.3,-11.3)$ & $13.4 \pm 2^{d}$ & 1.90 & 342 & 633707 \\
GS 1826-238 & $(9.3,-6.1)$ & $6.7^{c}$ & 3.00 & 347 & 87798 \\
GX 9 + 9 & $(8.5,9.0)$ & $4.4^{g}$ & 3.31 & 80 & 108956 \\
HETEJ1900.1-2455 & $(11.30,-12.87)$ & $5^{h}$ & 1.76 & 71 & 57691 \\
SAXJ1808.4-3658 & $(355.39,-8.15)$ & $2.8^{c}$ & 1.76 & 173 & 568390 \\
Serpens X-1 & $(36.12,4.84)$ & $11.1 \pm 1.6^{d}$ & 5.42 & 66 & 20858 \\
Swift J1753.5-0127 & $(24.89,12.18)$ & $8.42_{-2.85}^{+4.32}$ & 2.98 & 249 & 428804 \\
\hline
\end{tabular}

Notes. $N(\mathrm{HI})$ in units of $10^{21} \mathrm{~cm}^{-2}$.

Distances obtained from ${ }^{a}$ in't Zand et al. (2003); ${ }^{b}$ Wang \& Chakrabarty (2004);

${ }^{c}$ Galloway et al. (2008); ${ }^{d}$ Jonker \& Nelemans (2004); ${ }^{e}$ Kuulkers et al. (2003);

${ }^{f}$ Gomez, Mason \& Robinson (2015); ${ }^{g}$ Grimm, Gilfanov \& Sunyaev (2002); ${ }^{h}$ Hynes et al. (2004); ${ }^{i}$ Gandhi et al. (2019).

significance of the detection for a particular line (e.g. N VI K $\alpha$ detection). Tables 1 and 2 list the analysed Galactic and extragalactic sources, respectively, including the Galactic coordinates, H I column densities, which are taken from Willingale et al. (2013), total exposure times, and total number of counts of all observations in the $\mathrm{N} \mathrm{K}$-edge. The distances to the sources are included for all LMXBs. Observations were reduced using the Science Analysis System (SAS, ${ }^{1}$ version 18.0.0) including background subtraction and following the standard procedure to obtain high-resolution spectra from the Reflection Grating Spectrometers (RGS, den Herder et al. 2001). In particular, we follow the SAS thread to reduce RGS data and extract spectra of point-like sources. Using the rgsproc task, the procedure included the filtering of events and exposure, to exclude flaring background, and the creation of response matrices. Each spectrum was rebinned to have at least 1 count per channel.

For each source, all observations were fitted simultaneously using the XSPEC package (version 12.10.12) in the 24-32 A wavelength region. The continuum was modelled with a powerlaw*constant model, where the Photon-Index was set free to vary but tied among all the observations of the same source, while the constant accounts for differences in the normalization among them. Moreover, we used cash statistics (Cash 1979) in the spectral fitting analysis.

\subsection{Nitrogen photoabsorption cross-sections}

We use the K-edge photoionization cross-sections for $\mathrm{N}$ II-N V computed by García et al. (2009), which include detailed calculations of atomic properties of K-vacancy states for all ions of the nitrogen isonuclear sequence. Importantly, the smearing of the K-edge due to both Auger and radiation damping is taken into account. In the cases of N VI and N VII, we used the K-edge photoionization crosssections from the Opacity Project (Badnell et al. 2005). Fig. 1 shows the photoabsorption cross-sections included in the model, which includes the cold, warm, and hot phases of the ISM.

\footnotetext{
${ }^{1}$ https://www.cosmos.esa.int/web/xmm-newton/sas

${ }^{2}$ https://heasarc.gsfc.nasa.gov/xanadu/xspec/
}

We included these $\mathrm{N}$ photoabsorption cross-sections in a modified version of the ISMabs model (Gatuzz et al. 2015), thus allowing the column densities for the ionic species of interest to be free parameters in the data fitting. We fixed the H I ISMabs column densities to the values provided by Willingale et al. (2013). For each source, the column densities were linked between the different observations. We note that a detailed benchmarking of the main doublet/triplet resonance line positions (see Fig. 1) cannot be performed given that the differences in their relative positions are smaller than the instrumental spectral resolution. For example, for $\mathrm{N}$ II $\mathrm{K} \alpha$ and $\mathrm{N}$ III $\mathrm{K} \alpha$ we have a minimum separation for contiguous peaks of $\Delta \lambda \sim 22.1 \mathrm{~m} \AA$ and $\Delta \lambda \sim 31.2 \mathrm{~m} \AA$, respectively, while the RGS resolution in the $\mathrm{N}$ K-edge region is $\Delta \lambda \sim 60 \mathrm{~m} \AA$. Therefore, we used the atomic data as computed by García et al. (2009), which estimated the accuracy of the K-threshold to be within $1 \mathrm{eV}$.

\section{RESULTS FROM SPECTRAL FITS}

The best-fitting results are listed in Table 3. We have found acceptable fits, from the statistical point of view, although for most of the sources we have obtained upper limits for the relevant parameters. We labelled the multiple phases of the gaseous ISM as cold (NI), warm $(\mathrm{N}$ II $+\mathrm{N}$ III $)$, and hot $(\mathrm{N}$ V + N VI $+\mathrm{N}$ VII $)$. Fig. 2 shows the best-fitting column densities obtained. We note that for the cold component the column densities are systematically larger for the Galactic sources (black points) than for the extragalactic sources (red points). For the warm-hot components, on the other hand, the column densities tend to be similar between both types of sources. Fig. 3 shows the distribution of the cold, warm, and hot column densities as a function of the Galactic latitude. The plot shows that the cold-warm column densities decrease with the Galactic latitude, while the hot component does not appear to show any correlation, although for this case most of the results correspond to upper limits. This implies that the cold component is mostly concentrated in the Galactic disc, while the hot component is more homogeneously distributed. Previous studies suggest that a single disc model cannot fit the hot component of the ISM but requires a spherically symmetric 
Table 2. List of extragalactic observations analysed.

\begin{tabular}{|c|c|c|c|c|}
\hline Source & $\begin{array}{c}\text { Galactic } \\
\text { coordinates }\end{array}$ & $N(\mathrm{H} \mathrm{I})$ & $\begin{array}{l}\text { Exposure } \\
\text { time (ks) }\end{array}$ & $\begin{array}{l}\text { Number of counts } \\
\quad(24-32 \AA)\end{array}$ \\
\hline 1ES $1028+511$ & $(161.44 ; 54.44)$ & 1.26 & 300 & 61972 \\
\hline 1ES $1553+113$ & $(21.91 ; 43.96)$ & 4.35 & 2065 & 396199 \\
\hline 1H $0414+009$ & $(191.81 ;-33.16)$ & 13.70 & 91 & 12194 \\
\hline 1Н 0707-495 & $(260.17 ;-17.67)$ & 6.55 & 1395 & 127334 \\
\hline $1 \mathrm{H} 1219+301$ & $(186.36 ; 82.73)$ & 0.20 & 29 & 29318 \\
\hline $1 \mathrm{H} 1426+428$ & $(77.49 ; 64.90)$ & 1.14 & 425 & 145627 \\
\hline $3 \mathrm{C} 120$ & $(190.37 ;-27.40)$ & 19.30 & 305 & 19907 \\
\hline $3 \mathrm{C} 273$ & $(289.95 ; 64.36)$ & 1.78 & 1426 & 912126 \\
\hline 3C 279 & $(305.10 ; 57.06)$ & 2.22 & 153 & 10181 \\
\hline 3C 390.3 & $(111.44 ; 27.07)$ & 4.51 & 123 & 29626 \\
\hline Ark 120 & $(201.69 ;-21.13)$ & 1.40 & 773 & 203191 \\
\hline Ark 564 & $(92.14 ;-25.34)$ & 6.74 & 890 & 556839 \\
\hline ESO 141-G055 & $(338.18 ;-26.71)$ & 6.41 & 268 & 69813 \\
\hline ESO 198-G24 & $(271.64 ;-57.95)$ & 3.27 & 178 & 20460 \\
\hline Fairall 9 & $(295.07 ;-57.83)$ & 3.43 & 328 & 42360 \\
\hline H2356-309 & $(12.84 ;-78.04)$ & 1.48 & 702 & 91678 \\
\hline $\mathrm{H} 1821+643$ & $(94.00 ; 27.42)$ & 0.39 & 121 & 22990 \\
\hline HE1143-1810 & $(281.85 ; 41.71)$ & 0.34 & 184 & 51334 \\
\hline IRAS13349+2438 & $(20.60 ; 79.32)$ & 1.07 & 199 & 24152 \\
\hline IC4329A & $(317.50 ; 30.92)$ & 0.56 & 165 & 19327 \\
\hline IZw1 & $(123.75 ;-50.17)$ & 6.01 & 382 & 50616 \\
\hline MCG-6-30-15 & $(313.29 ; 27.68)$ & 0.47 & 794 & 341504 \\
\hline MR2251-178 & $(46.20 ;-61.33)$ & 2.67 & 592 & 131180 \\
\hline Mrk 279 & $(115.04 ; 46.86)$ & 1.72 & 188 & 74129 \\
\hline Mrk 421 & $(179.83 ; 65.03)$ & 2.01 & 3004 & $7.37 \times 10^{6}$ \\
\hline Mrk 501 & $(63.60 ; 38.86)$ & 1.66 & 200 & 109344 \\
\hline Mrk 509 & $(35.97 ;-29.86)$ & 5.04 & 835 & 498007 \\
\hline Mrk 766 & $(190.68 ; 82.27)$ & 0.19 & 739 & 223087 \\
\hline Mrk 841 & $(11.21 ; 54.63)$ & 2.43 & 206 & 35132 \\
\hline NGC 3783 & $(287.46 ; 22.95)$ & 13.80 & 486 & 46906 \\
\hline NGC 4593 & $(297.48 ; 57.40)$ & 2.04 & 386 & 97579 \\
\hline NGC 5548 & $(31.96 ; 70.50)$ & 1.69 & 1025 & 108103 \\
\hline NGC 7213 & $(349.59 ;-52.58)$ & 1.12 & 181 & 22796 \\
\hline NGC 7469 & $(83.10 ;-45.47)$ & 5.24 & 857 & 229214 \\
\hline PG1116+215 & $(223.36 ; 68.21)$ & 1.43 & 393 & 49771 \\
\hline PKS 0548-32 & $(237.57 ;-26.14)$ & 2.87 & 254 & 58464 \\
\hline PKS 0558-504 & $(257.96 ;-28.57)$ & 4.18 & 933 & 420921 \\
\hline PKS 2005-489 & $(350.37 ;-32.60)$ & 4.66 & 253 & 22609 \\
\hline PKS 2155-304 & $(17.73 ;-52.25)$ & 1.63 & 2000 & $2.53 \times 10^{6}$ \\
\hline Tons 180 & $(138.99 ;-85.07)$ & 1.54 & 222 & 42199 \\
\hline
\end{tabular}

Note. $N(\mathrm{HI})$ in units of $10^{20} \mathrm{~cm}^{-2}$

profile, to account for the Galactic halo contribution (Miller \& Bregman 2013, 2015; Nicastro et al. 2016b; Gatuzz \& Churazov 2018).

The presence of absorption features due to material intrinsic to the source (e.g. warm absorbers in active galactic nuclei) may lead to misidentification of certain absorption lines. To study such intrinsic absorber we used the warmabs model, which is computed with the XSTAR photoionization code (Kallman \& Bautista 2001). This model considers the physical conditions for an ionizing source surrounded by a gas and takes into account physical processes such as photoionization, dielectronic and radiative recombination, excitation and electron impact collisional ionization. The model assumes ionization equilibrium conditions, a Maxwellian electron velocity distribution, and that the gas responsible for emission and absorption has a uniform temperature and ionization throughout. The parameters of the model includes the column density of the absorber
$(N \mathrm{HI})$, the ionization parameter $(\log \xi)$, elemental abundances $(\mathrm{A} x)$ broadening turbulence $\left(v_{\text {turb }}\right)$, and redshift $(z)$.

We have tested the effects in the ISMabs column densities when including a warmabs component in addition to the ISMabs components, for Ark 564. In order to account for variations in the spectral energy distribution (SED), which affects the photoionization rate, we fitted the warmabs $N(\mathrm{HI})$ and $\log \xi$ parameters independently for each observation, while the ISMabs column densities were linked between the different observations (i.e. to account for the ISM contribution). We have found that the warmabs best fit prefers a highly ionized component, but the warmabs column densities for such component are poorly constrained. Moreover, while the cold and warm ISMabs column densities obtained are not affected by the inclusion of warmabs, the uncertainties in IS Mabs column densities for the hot component increase significantly. Similar results were obtained for other sources with large number 

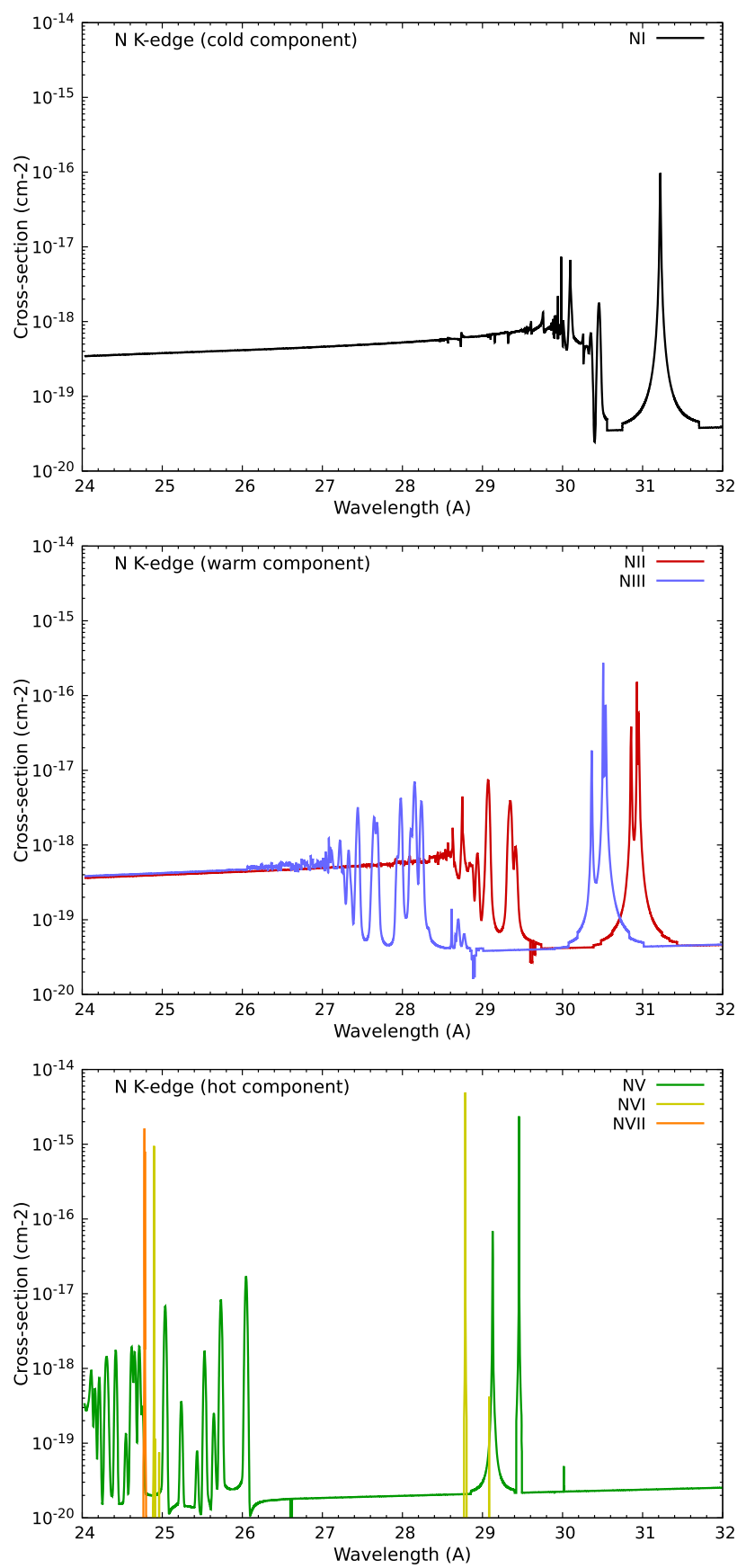

Figure 1. N I (top panel), N II, N III (middle panel), N v, N VI, and N VII (bottom panel) photoabsorption cross-sections computed by García et al. (2009) and included in the model.

of counts, including 1ES1028+511, 1ES1553+113, 1H0414+009, and $1 \mathrm{H} 0707-495$. It is important to note that the fits here presented are done within a small wavelength region (24-32 ̊). In order to perform a more detailed analysis of the absorber associated with the sources, a complete analysis of the RGS data is desired to identified absorption features in the spectra due to different ions, apart from nitrogen. However, such analysis is beyond the scope of this work. Finally, it is important to note that when fitting independently the intrinsic absorber for different observations, the computation time to perform error calculation increases exponentially (e.g. there are 37 observations for Mrk 421).

\subsection{Comparison with previous works}

We have collected N I column density values from previous studies. Meyer, Cardelli \& Sofia (1997, MEY + 97) analysed the interstellar N I $\lambda \lambda$ 1160,1161 absorption doublet towards eight stars using the Hubble Space Telescope Goddard High Resolution Spectrograph (GHRS). Moos et al. (2002, MOO + 02) determined N I column densities along seven lines of sight using N I $\lambda 1160$ observations obtained with the Far Ultraviolet Spectroscopic Explorer (FUSE). Knauth, Meyer \& Lauroesch $(2006$, KNA + 06) reported on the analysis of 13 stars using FUSE observations. Finally, Gudennavar et al. (2012, GUD + 12) created a data base of multiple interstellar column densities using absorption line data towards 3008 stars, 164 of them with $\mathrm{N}$ I values.

Fig. 4 shows the distribution of N I values as a function of Galactic latitude (top panel) and distance (bottom panel) including the collected sample. In the case of the distances, we have not included the LMXB $4 \mathrm{U} 1957+11$ due to its large uncertainty. For the cold ISM component, it is expected to show a strong correlation between the column densities and the Galactic latitude (see for example Gatuzz \& Churazov 2018). For the cold component of the ISM, it is commonly assumed in the literature to be exponentially decreasing perpendicular to the Galactic plane, with larger column densities near the Galactic centre (see e.g. Robin et al. 2003; Kalberla \& Kerp 2009). For the X-ray data we have included only those Galactic sources for which N I is well constrained (i.e. no upper limits. See Table 3). We found that the X-ray column densities derived from the spectral fitting are in good agreement with previous results, with a clear tendency to decrease as we move away from the Galactic plane $\left(\mid\right.$ Latitude $\left.\mid>50^{\circ}\right)$ with variations in regions that form stars more actively than others (e.g. spiral arm regions such as Vela or Orion). However, the number of lines of sight does not allow to cover small-scale structure (i.e. au) but rather large-scale. Finally, it is important to note that the hot component is only accessible through UV/X-ray observations.

\subsection{ISM structure}

Previous analysis of the ISM multiphase structure using X-ray absorption technique has shown that the gas physical state is dominated by the cold component, with mass fractions for the ISM phases in the Galactic disc of cold $\sim 90$ per cent, warm $\sim 8$ per cent, and hot $\sim 2$ per cent components (e.g. Yao \& Wang 2006; Piontek \& Ostriker 2007; Pinto et al. 2013; Gatuzz \& Churazov 2018). The uncertainties in the ISMabs column densities obtained prevent us to compute accurate mass fractions for all sources. For example, we noted that our best-fitting results show a very large contribution of the N VII column density associated with the hot component (e.g. GX9+9, 3C 273, MCG-6-30-15, Mrk766, PKS2005-489), while previous work indicates that the hot phase represent less than 1 per cent of the total ISM (e.g. Pinto et al. 2013). However, for these sources our analysis show that the rest of ionic species are not well constrained. Moreover, our ISMabs model includes the column densities as free parameters in the model and therefore we do not consider any ionization balance for the nitrogen ionic species. In this sense, a proper definition requires a spectral fitting with a more complete physical model that compute ionization balance depending on temperature. The contribution of the different ISM phases depends on the location of the absorber. While the density distribution for the cold-warm components is typically modelled with an exponential profile for the Galactic disc, the hot component includes the contribution of the Galactic halo 
Table 3. Best-fitting nitrogen column densities obtained.

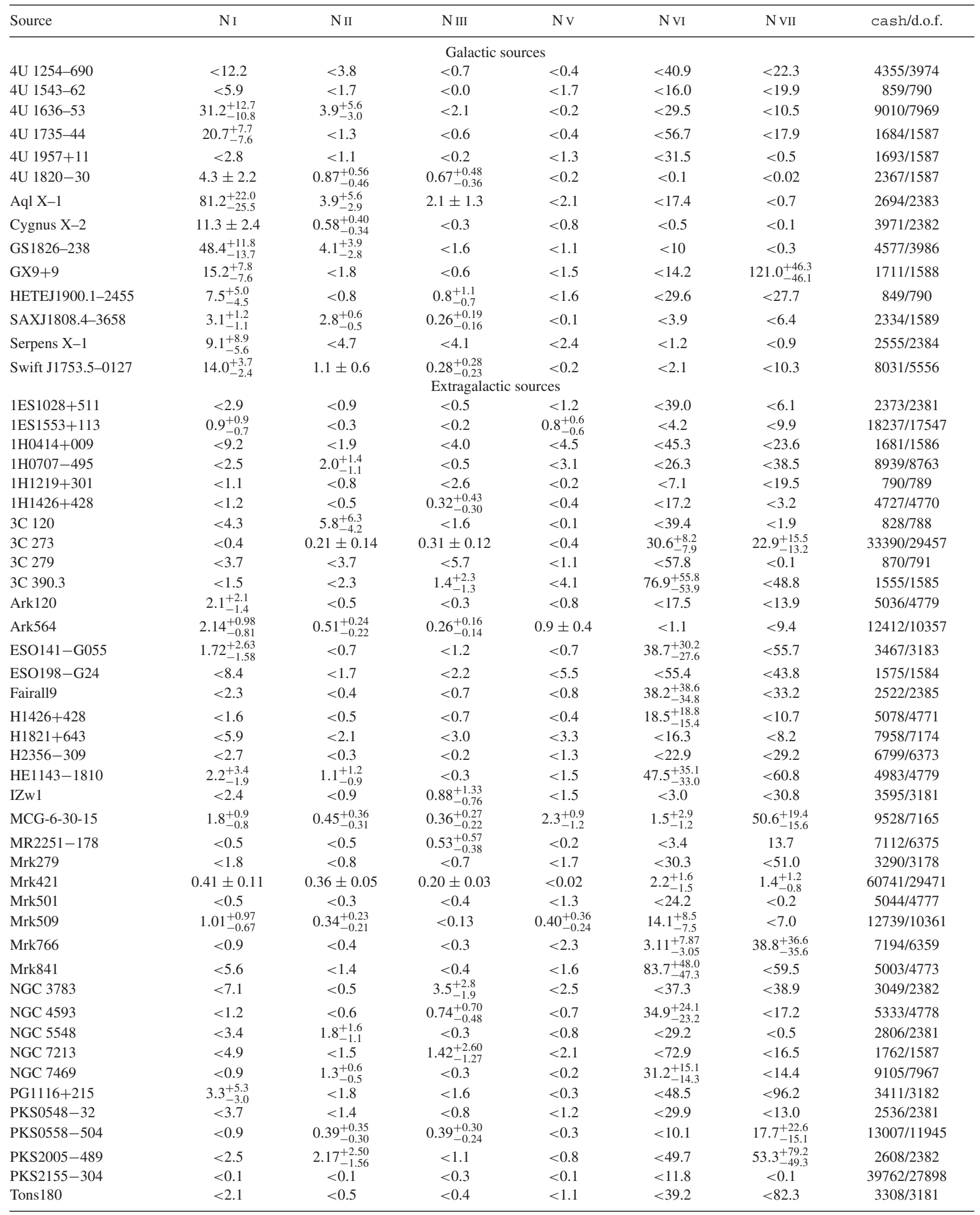

Note. Column densities in units of $10^{16} \mathrm{~cm}^{-2}$. 

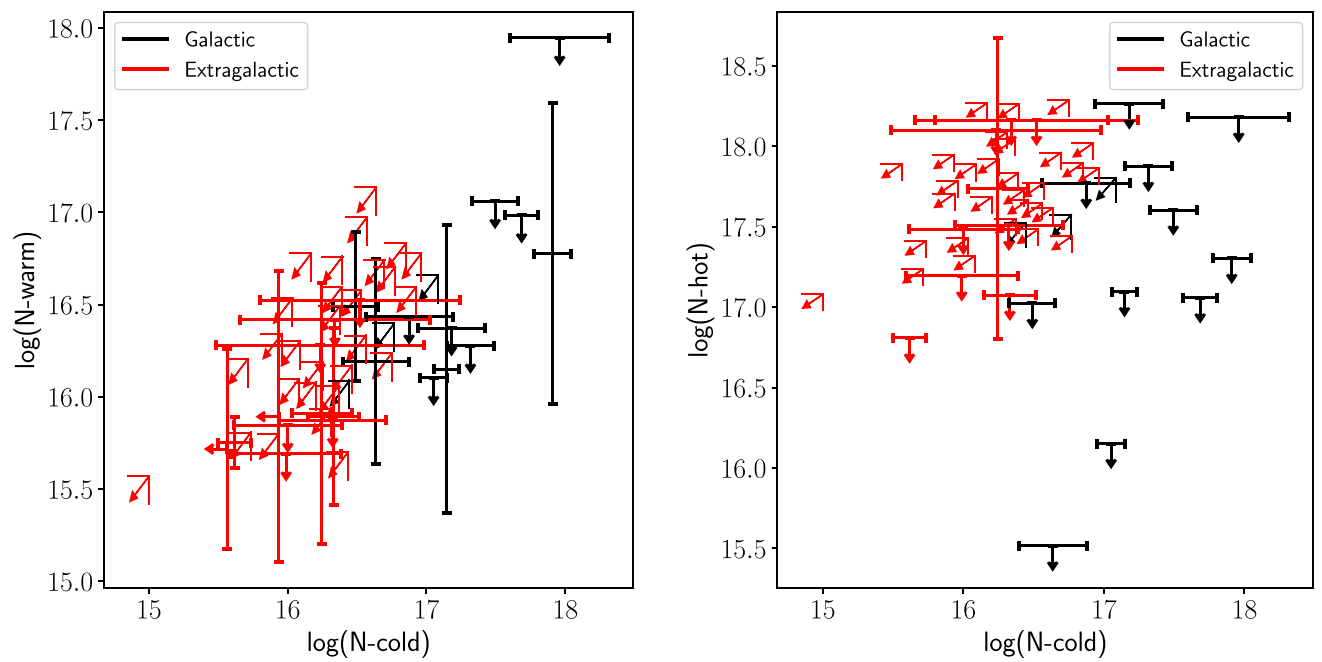

Figure 2. Best-fitting column densities for the cold (N I), warm ( $\mathrm{N}$ II $+\mathrm{N}$ III), and hot ( $\mathrm{N}$ V $+\mathrm{N}$ VI $+\mathrm{N}$ VII) ISM phases. The black data points correspond to LMXB, while the red points correspond to extragalactic sources.

(e.g. Miller \& Bregman 2013, 2015; Nicastro et al. 2016b; Gatuzz \& Churazov 2018). However, the absence of sources in our sample near the Galactic centre, a region heavily affected by the cold gas absorption, does not allow us to constrain the density profiles of the different ISM phases. More information about the physical properties of the gas (e.g. temperature and abundances) will be included in future works, with the inclusion of more complex models (e.g. warmabs).

\subsection{Future prospects}

Future X-ray high-resolution spectra mission, such as Arcus (Smith et al. 2016) and Athena (Nandra et al. 2013), will benefit greatly from this atomic data benchmarking. For example, Fig. 5 shows a $10 \mathrm{ks}$ simulation for an extragalactic source (i.e. Mrk 421 in high-state) obtained with Athena for the baseline configuration. $^{3}$ The position for the main absorption lines are indicated. The plot shows the outstanding capabilities of the instrument, with the main resonance absorption lines visible byeye, compared with the RGS spectra analysed in this paper. For such observation the line profiles can be studied with an accuracy at the percent level. More important, by measuring simultaneously multiple resonance lines (e.g. $\mathrm{K} \alpha$ and $\mathrm{K} \beta$ ) for the same ions, model-independent constraint on the broadening will be obtained.

\section{CONCLUSIONS}

We have carried out analysis of the ISM nitrogen K-edge absorption (24-32 ̊) using high-resolution XMM-Newton spectra. Our data sample consist of $12 \mathrm{LMXBs}$ and 40 extragalactic sources. For each source we fitted all observations simultaneously using a powerlaw*constant model for the continuum and a modified version of the ISMabs model for the spectral absorption features. We have found acceptable fits, from the statistical point of view, for most of sources. We have measured column densities for $\mathrm{N} \mathrm{I}$,

\footnotetext{
${ }^{3}$ http://x-ifu-resources.irap.omp.eu/public/responses/cc_configuration/
}

$\mathrm{N}$ II, $\mathrm{N}$ III, $\mathrm{N}$ V, $\mathrm{N}$ VI, and $\mathrm{N}$ VII ionic species, which trace the cold, warm, and hot phases of the ISM. For the cold component we have found that the distribution of the column density distribution as a function of Galactic latitude is in good agreement with UV measurements. For the hot component we have not found such correlation, most likely due to the contribution from the Galactic halo. We have tested the effects in the ISMabs column densities when including a warmabs component, to account for absorption intrinsic to the sources. For such test, we link the ISM column densities between different observations (to account for the ISM contribution), while the warmabs $N(\mathrm{HI})$ and $\log \xi$ parameters were fitted independently for each observation. We have found that both cold and warm ISMabs column densities obtained are not affected by the inclusion of the warmabs component. However, the uncertainties in the hot ISM phase increase significantly, while the column densities for the warmabs are not constrained. Such analysis point out the importance of modelling simultaneously multiple $\mathrm{K}$ edge absorption regions (e.g. $\mathrm{O}, \mathrm{Ne}, \mathrm{Mg}$ ) in order to study the contribution from both, the local ISM and intrinsic absorption. Future observations with new-generation instrumentation such as Arcus and Athena will allow a finer examination of the N K-edge structure.

\section{ACKNOWLEDGEMENTS}

We thank the anonymous referee for the careful reading of our manuscript and the valuable comments. JAG acknowledges support from the Smithsonian Astrophysical Observatory grant AR021003X, and from the Alexander von Humboldt Foundation.

\section{DATA AVAILABILITY}

Observations analysed in this article are available in the XMMNewton Science Archive (XSA) (http://nxsa.esac.esa.int/nxsa-web /\#search). The ISMabs model is included in the XSPEC data analysis software (https://heasarc.gsfc.nasa.gov/xanadu/xspec/). 

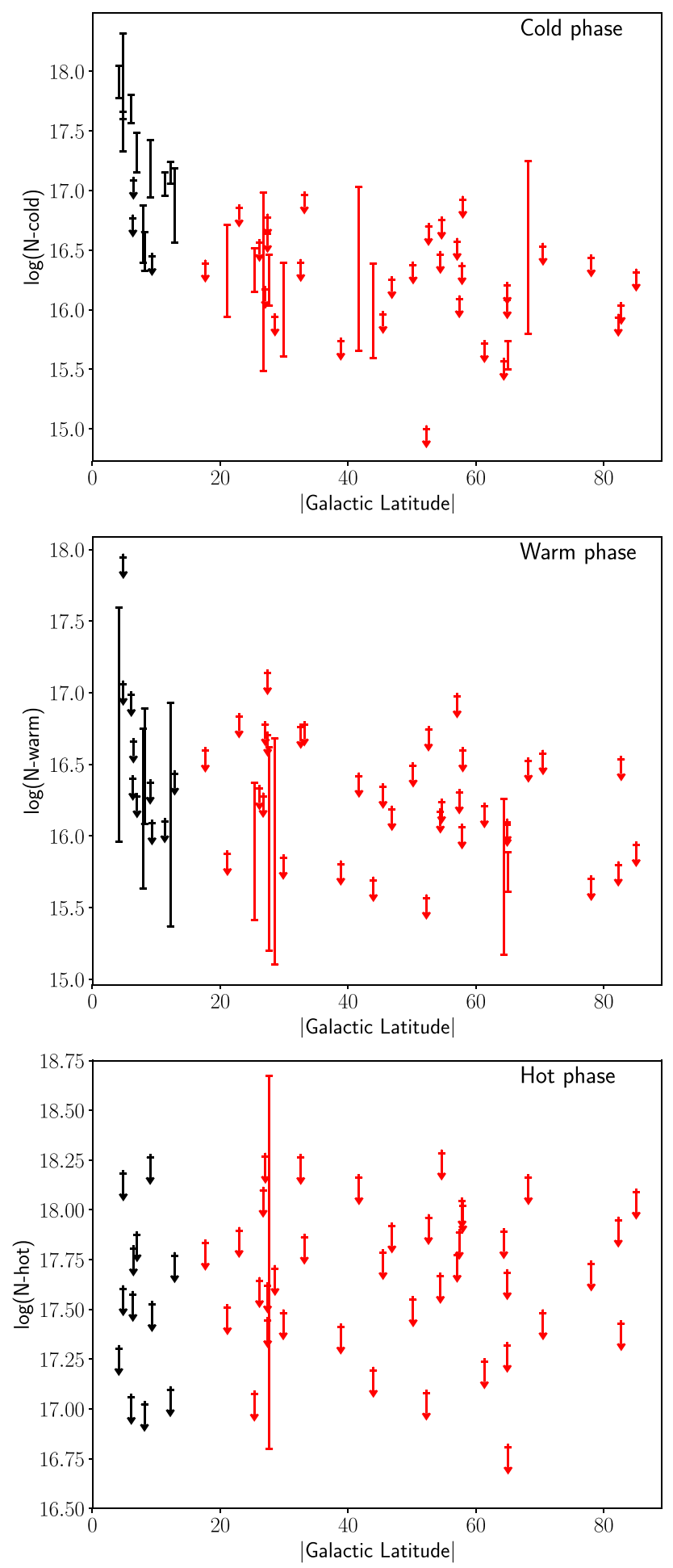

Figure 3. Best-fitting column densities for the cold (N I), warm (N II + N III), and hot $(\mathrm{N} v+\mathrm{N}$ VI $+\mathrm{N}$ VII $)$ ISM phases as a function of the Galactic latitude. The black data points correspond to LMXB, while the red points correspond to extragalactic sources.
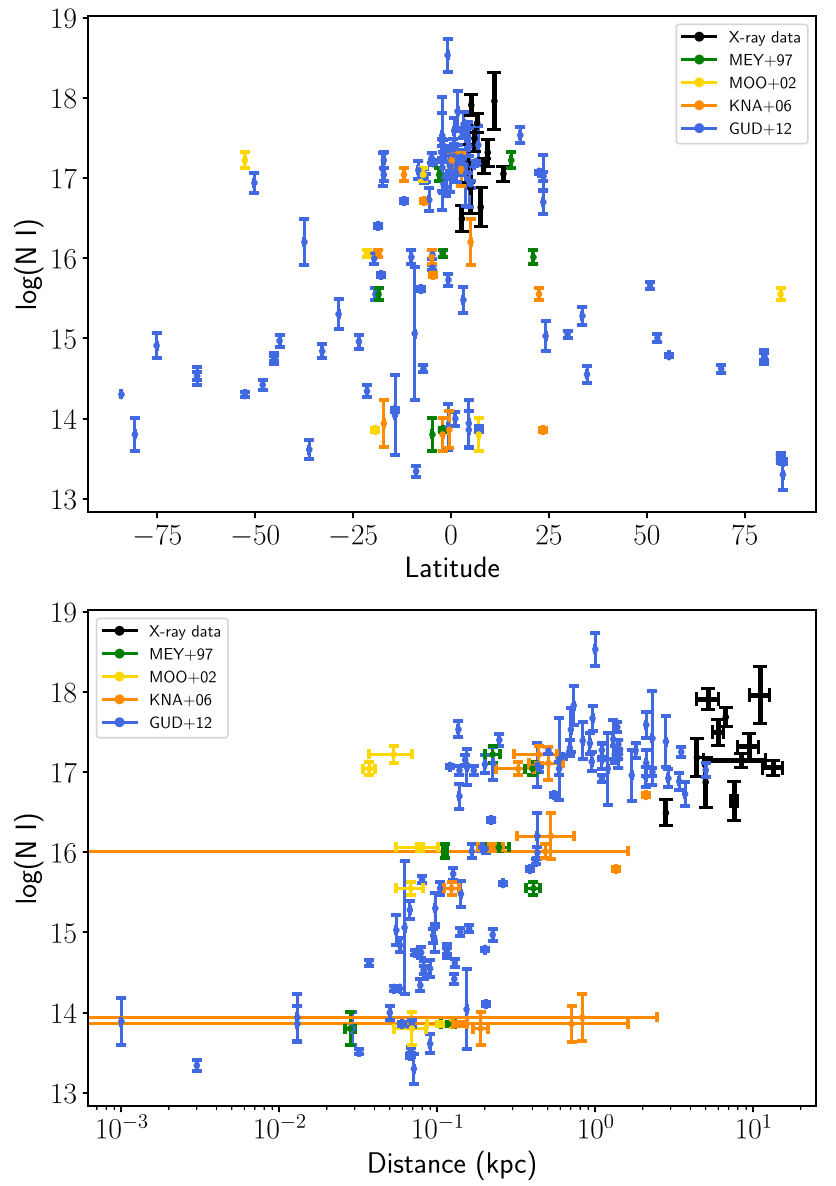

Figure 4. Top panel: $\mathrm{N}$ I column densities distribution as a function of Galactic latitude for different Galactic samples. Bottom panel: N I column densities distribution as a function of the distance.

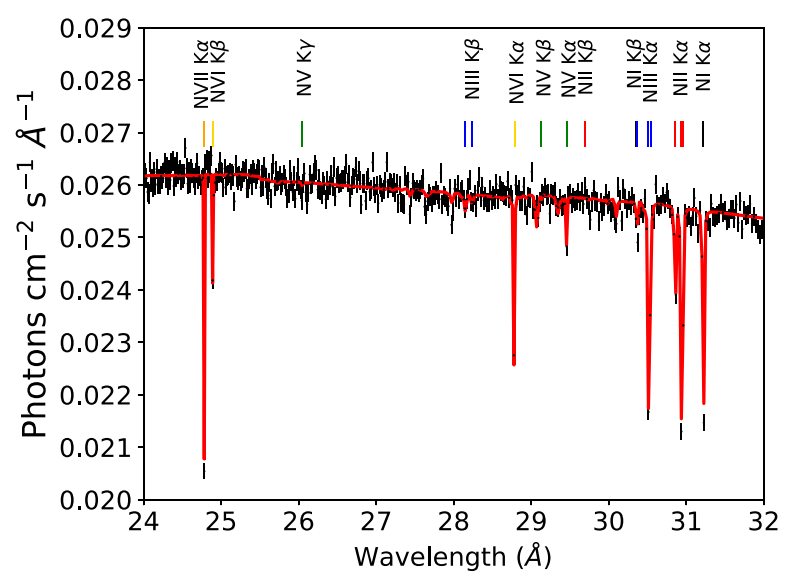

Figure 5. Athena simulation of the N K-edge photoabsorption region for an extragalactic source (e.g. Mrk 421). The total exposure time is $10 \mathrm{ks}$ and the flux is $\log F_{x}=-9.77 \mathrm{erg} \mathrm{cm}^{-2} \mathrm{~s}^{-1}$ in the $24-32 \AA$ wavelength range. 


\section{REFERENCES}

Badnell N. R., Bautista M. A., Butler K., Delahaye F., Mendoza C., Palmeri P., Zeippen C. J., Seaton M. J., 2005, MNRAS, 360, 458

Cash W., 1979, ApJ, 228, 939

den Herder J. W. et al., 2001, A\&A, 365, L7

Draine B. T., 2011, Physics of the Interstellar and Intergalactic Medium. Princeton Univ. Press, Princeton, NJ

Falgarone E., Verstraete L., Pineau Des Forêts G., Hily-Blant P., 2005, A\&A, 433, 997

Galloway D. K., Muno M. P., Hartman J. M., Psaltis D., Chakrabarty D., 2008, ApJS, 179, 360

Gandhi P., Rao A., Johnson M. A. C., Paice J. A., Maccarone T. J., 2019, MNRAS, 485, 2642

García J. et al., 2009, ApJS, 185, 477

García J., Mendoza C., Bautista M. A., Gorczyca T. W., Kallman T. R., Palmeri P., 2005, ApJS, 158, 68

Gatuzz E. et al., 2013a, ApJ, 768, 60

Gatuzz E. et al., 2013b, ApJ, 778, 83

Gatuzz E., Churazov E., 2018, MNRAS, 474, 696

Gatuzz E., García J., Kallman T. R., Mendoza C., Gorczyca T. W., 2015, ApJ, 800,29

Gatuzz E., Ness J.-U., Gorczyca T. W., Hasoglu M. F., Kallman T. R., García J. A., 2018a, MNRAS, 479, 2457

Gatuzz E., Rezaei K. S., Kallman T. R., Kreikenbohm A., Oertel M., Wilms J., García J. A., 2018b, MNRAS, 479, 3715

Gatuzz E., Gorczyca T. W., Hasoglu M. F., Schulz N. S., Corrales L., Mendoza C., 2020, MNRAS, 498, L20

Gomez S., Mason P. A., Robinson E. L., 2015, ApJ, 809, 9

Gorczyca T. W. et al., 2013, ApJ, 779, 78

Grimm H.-J., Gilfanov M., Sunyaev R., 2002, A\&A, 391, 923

Gudennavar S. B., Bubbly S. G., Preethi K., Murthy J., 2012, ApJS, 199, 8

Gupta A., Mathur S., Krongold Y., 2017, ApJ, 836, 243

Hasoğlu M. F., Abdel-Naby S. A., Gatuzz E., García J., Kallman T. R., Mendoza C., Gorczyca T. W., 2014, ApJS, 214, 8

Hynes R. I., Steeghs D., Casares J., Charles P. A., O’Brien K., 2004, ApJ, 609,317

in't Zand J. J. M., Kuulkers E., Verbunt F., Heise J., Cornelisse R., 2003, A\&A, 411, L487

Jenkins E. B., Tripp T. M., 2011, ApJ, 734, 65

Jonker P. G., Nelemans G., 2004, MNRAS, 354, 355

Juett A. M., Schulz N. S., Chakrabarty D., 2004, ApJ, 612, 308

Juett A. M., Schulz N. S., Chakrabarty D., Gorczyca T. W., 2006, ApJ, 648, 1066

Kalberla P. M., Kerp J., 2009, ARA\&A, 47, 27

Kallman T., Bautista M., 2001, ApJS, 133, 221
Knauth D. C., Meyer D. M., Lauroesch J. T., 2006, ApJ, 647, L115

Kuulkers E., den Hartog P. R., in't Zand J. J. M., Verbunt F. W. M., Harris W. E., Cocchi M., 2003, A\&A, 399, 663

Liao J.-Y., Zhang S.-N., Yao Y., 2013, ApJ, 774, 116

Luo Y., Fang T., 2014, ApJ, 780, 170

McKee C. F., Ostriker J. P., 1977, ApJ, 218, 148

Meyer D. M., Cardelli J. A., Sofia U. J., 1997, ApJ, 490, L103

Miller M. J., Bregman J. N., 2013, ApJ, 770, 118

Miller M. J., Bregman J. N., 2015, ApJ, 800, 14

Moos H. W. et al., 2002, ApJS, 140, 3

Nandra K. et al., 2013, preprint (arXiv:1306.2307)

Ness J. U. et al., 2011, ApJ, 733, 70

Ness J.-U. et al., 2003, ApJ, 594, L127

Nicastro F., Senatore F., Gupta A., Guainazzi M., Mathur S., Krongold Y., Elvis M., Piro L., 2016a, MNRAS, 457, 676

Nicastro F., Senatore F., Krongold Y., Mathur S., Elvis M., 2016b, ApJ, 828, L12

Pinto C., Kaastra J. S., Costantini E., Verbunt F., 2010, A\&A, 521, A79

Pinto C., Kaastra J. S., Costantini E., de Vries C., 2013, A\&A, 551, A25

Piontek R. A., Ostriker E. C., 2007, ApJ, 663, 183

Ramírez J. M., Komossa S., Burwitz V., Mathur S., 2008, ApJ, 681, 965

Robin A. C., Reylé C., Derrière S., Picaud S., 2003, A\&A, 409, 523

Smith R. K. et al., 2016, in den Herder J.-W. A., Takahashi T., Bautz M., eds, Proc. SPIE Conf. Ser. Vol. 9915, Space Telescopes and Instrumentation 2016: Ultraviolet to Gamma Ray. SPIE, Bellingham, p. 99054M

Smith R. A. N., Page M. J., Branduardi-Raymont G., 2007, A\&A, 461, 135

Stanimirović S., Zweibel E. G., 2018, ARA\&A, 56, 489

Steenbrugge K. C. et al., 2005b, A\&A, 434, 569

Steenbrugge K. C., Kaastra J. S., Sako M., Brand uardi-Raymont G., Behar E., Paerels F. B. S., Blustin A. J., Kahn S. M., 2005a, A\&A, 432, 453

Wang Z., Chakrabarty D., 2004, ApJ, 616, L139

Willingale R., Starling R. L. C., Beardmore A. P., Tanvir N. R., O’Brien P. T., 2013, MNRAS, 431, 394

Yao Y., Wang Q. D., 2006, ApJ, 641, 930

\section{APPENDIX: SPECTRA OF INDIVIDUAL SOURCE AND BEST-FITTING MODELS}

Figs A1 and A2 show the best-fitting models to the spectra for the individual LMXBs and extragalactic sources, respectively. For each source, all observations were combined for illustrative purposes. The black points correspond to the observations and the red lines indicate the best-fitting model. Residuals, in units of (data - model)/error, are included. The positions of the $\mathrm{K} \alpha$ and $\mathrm{K} \beta$ resonance lines are indicated for each ion, following the colour code used in Fig. 1. 

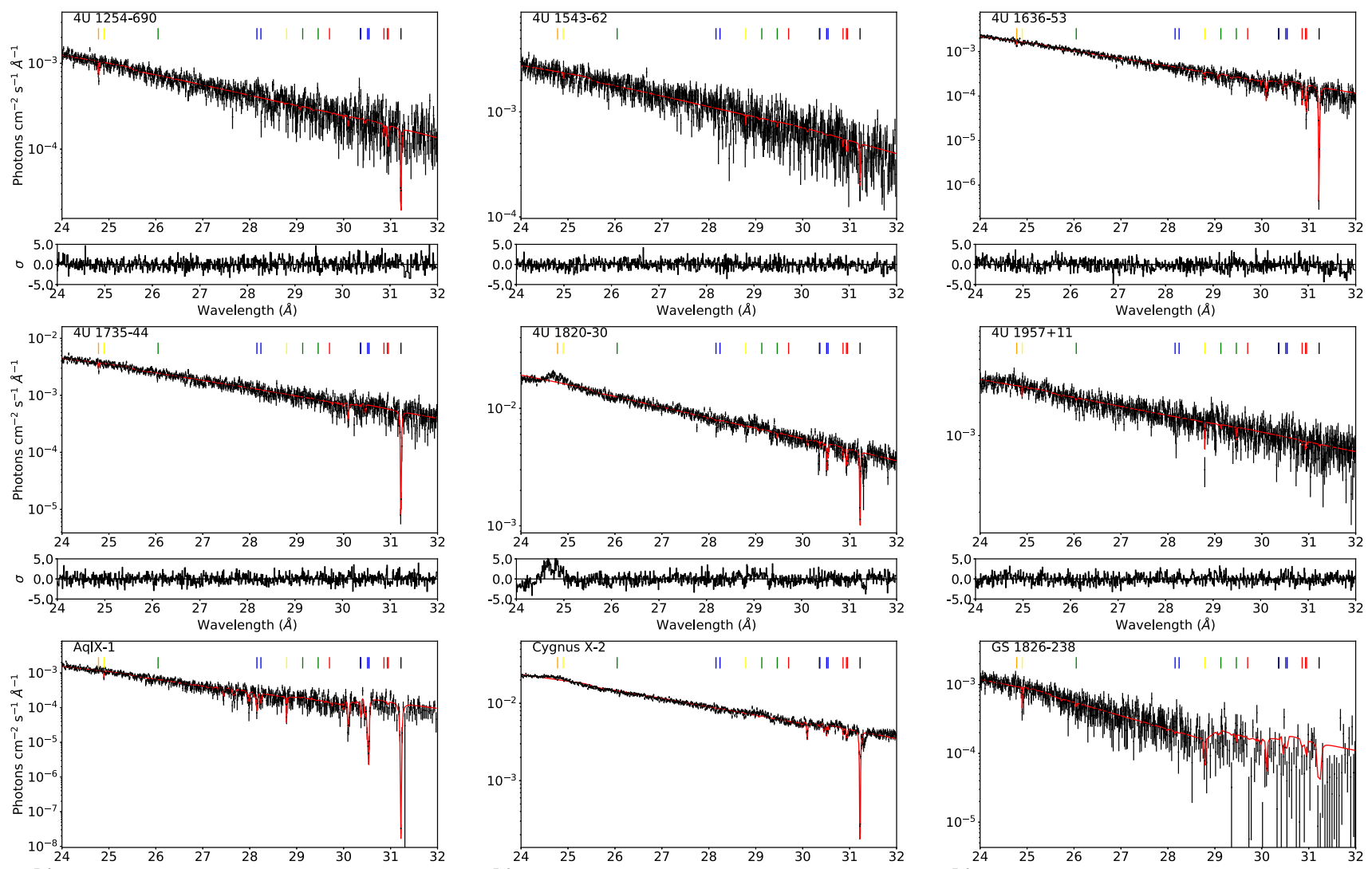

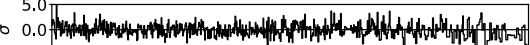
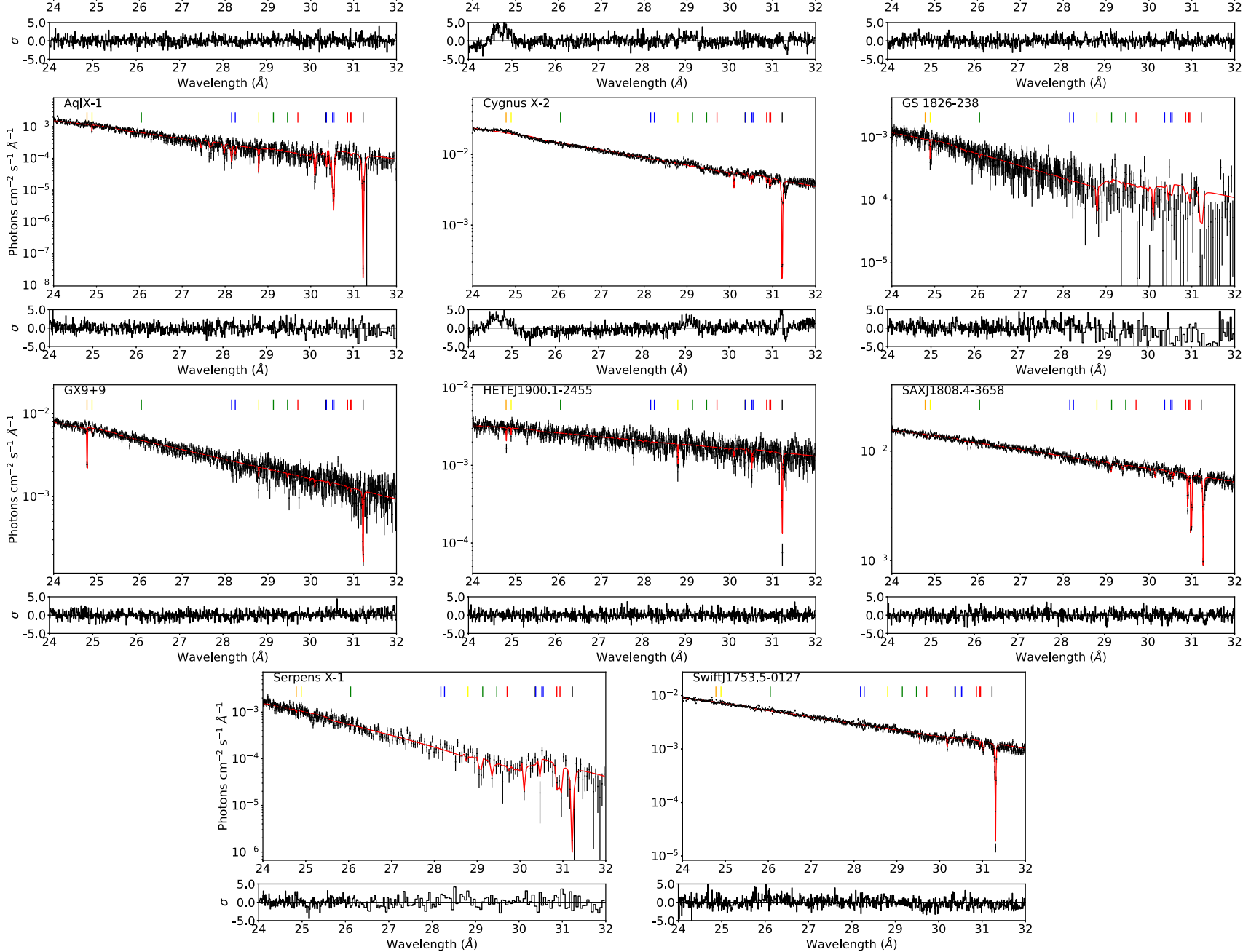

Figure A1. Best-fitting results in the N K-edge photoabsorption region for the LMXBs sample described in Table 1. In each panel, the black data points are the observations, while the solid red lines correspond to the best-fitting models. 


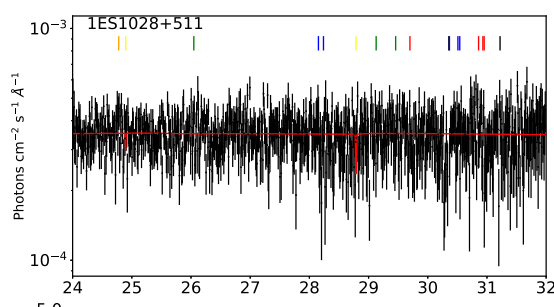

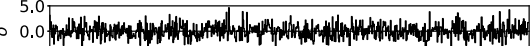

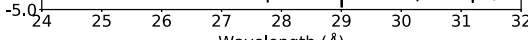
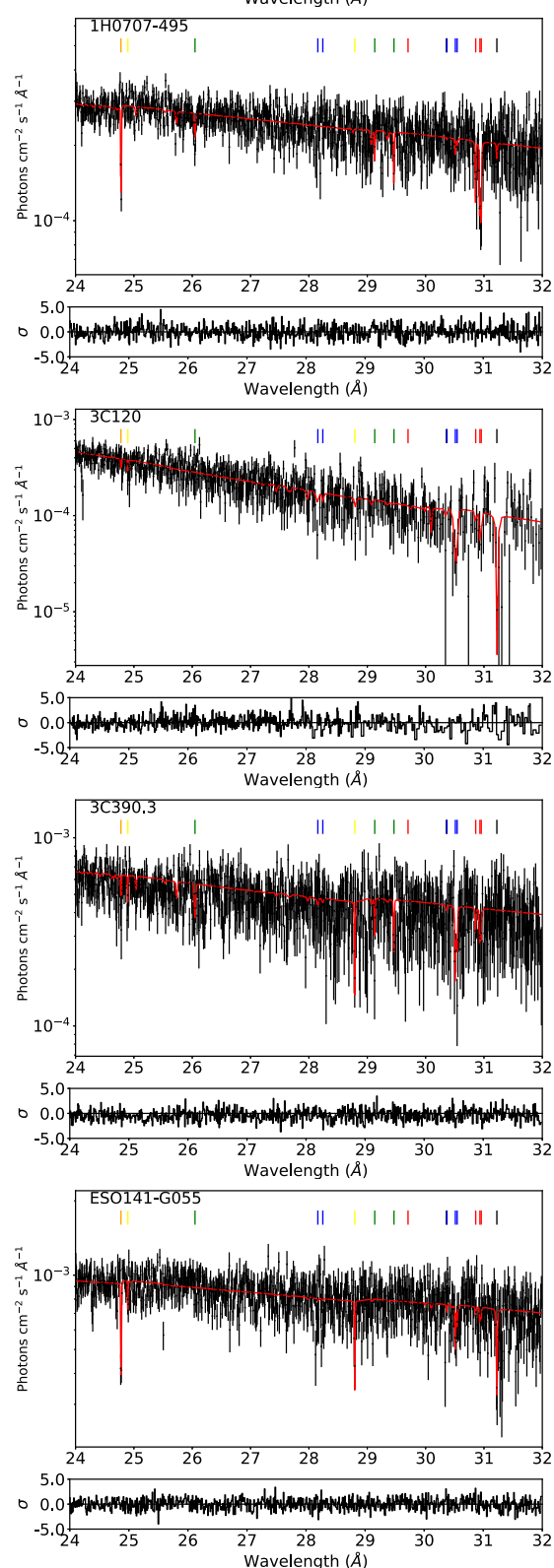

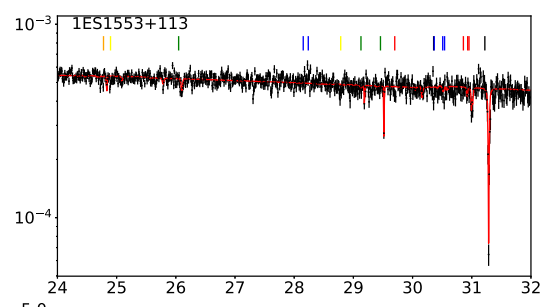

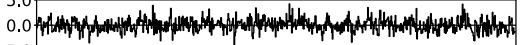
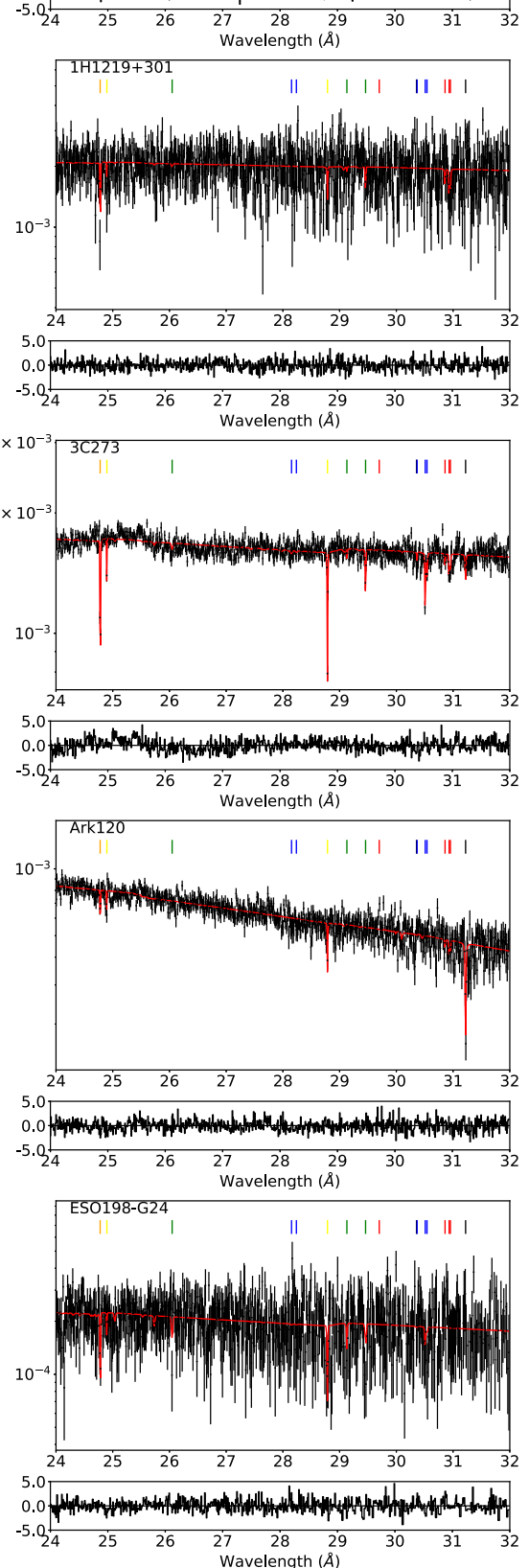

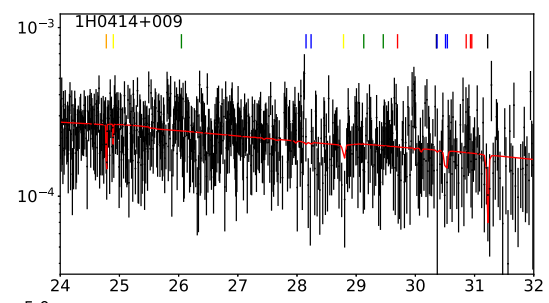

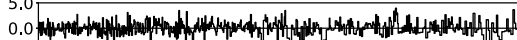
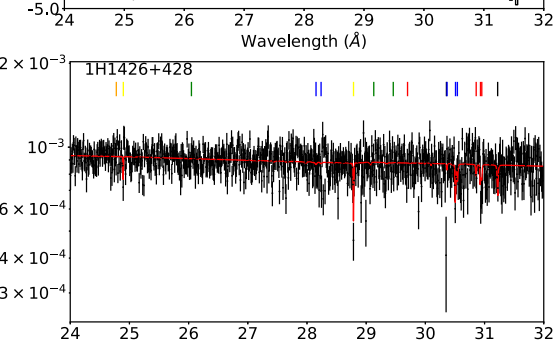

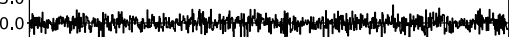
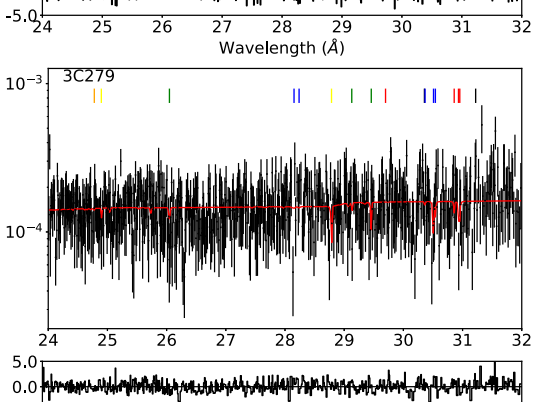

$\begin{array}{lllllllll}-5.04 & 25 & 26 & 27 & 28 & 29 & 30 & 31 & 32\end{array}$
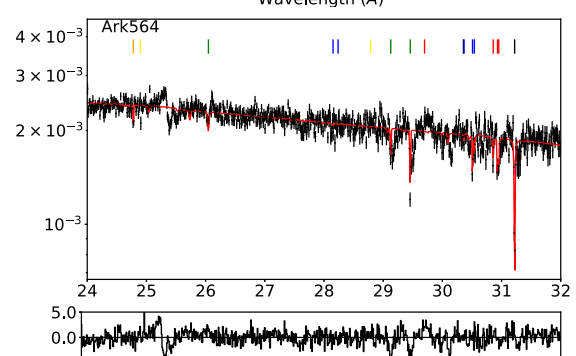

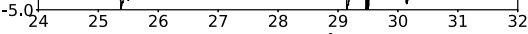

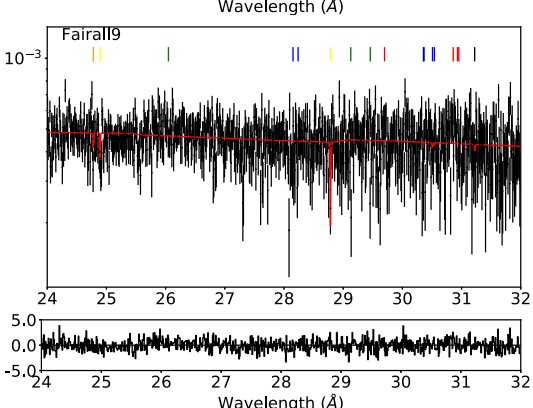

Figure A2. Best-fitting results in the N K-edge photoabsorption region for the Extragalactic sample described in Table 2. In each panel, the black data points are the observations and the solid red lines correspond to the best-fitting models. 

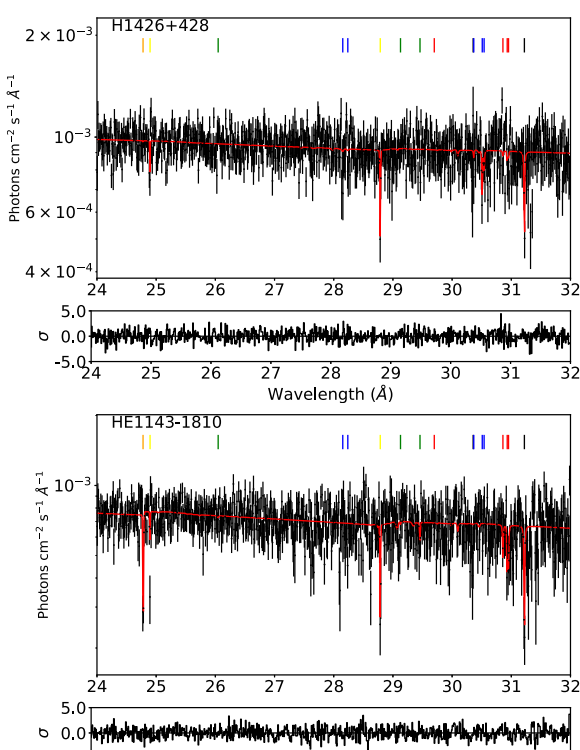

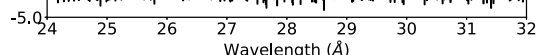

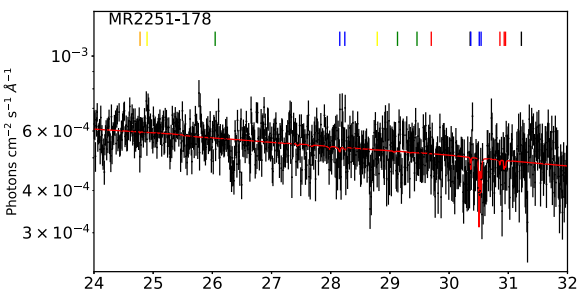

$\begin{array}{ccccccccc}24 & 25 & 26 & 27 & 28 & 29 & 30 & 31 & 32 \\ 0 & 0.0\end{array}$

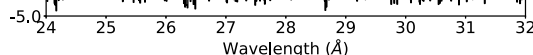

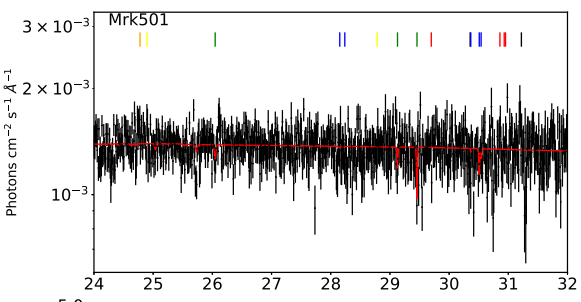

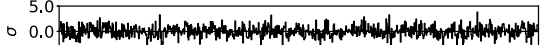

$\begin{array}{lllllllll}-5.0 & 25 & 26 & 27 & 28 & 29 & 30 & 31 & 32\end{array}$

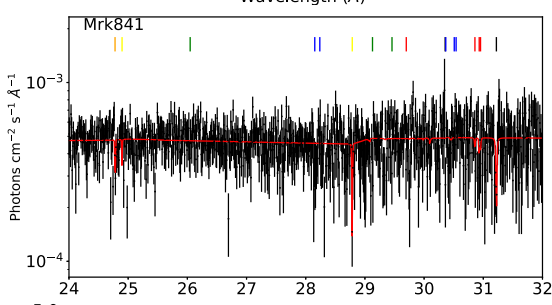

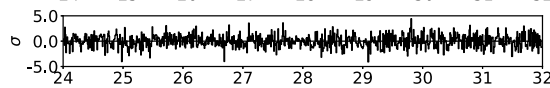
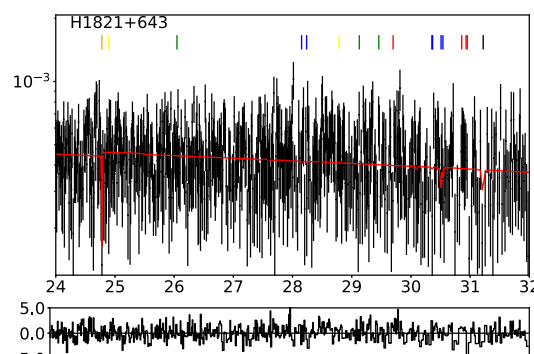

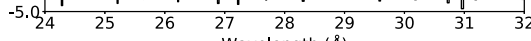
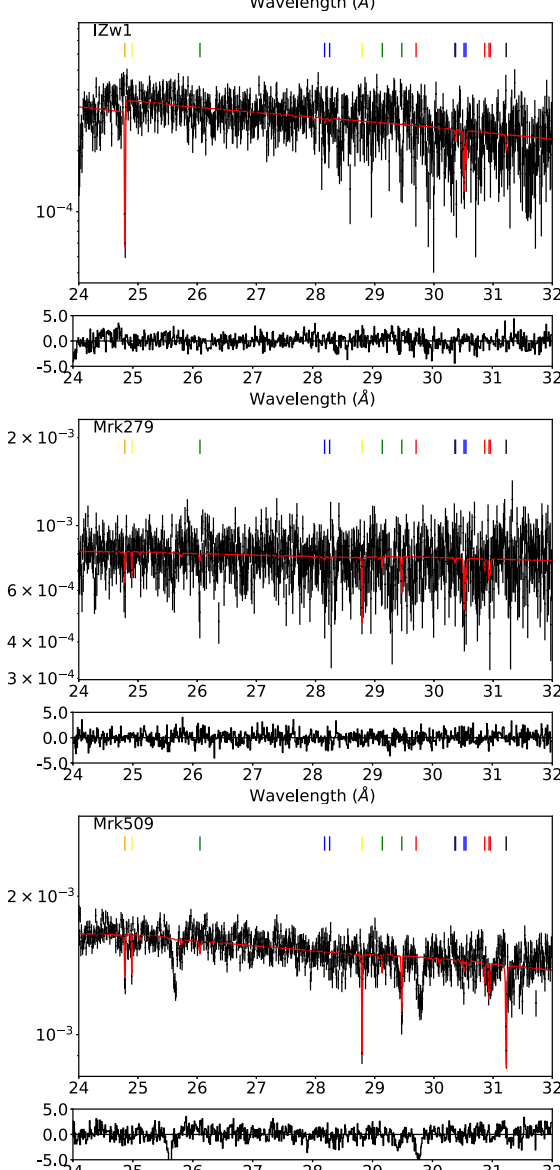

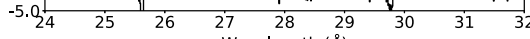

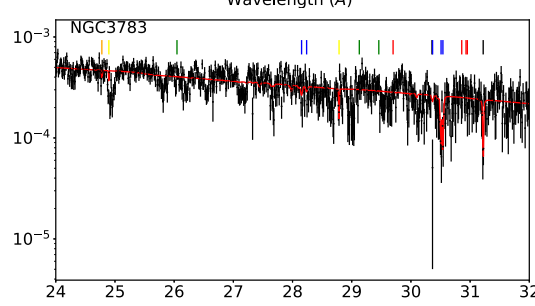

$\begin{array}{lllllllll}24 & 25 & 26 & 27 & 28 & 29 & 30 & 31 & 32 \\ 0.0\end{array}$

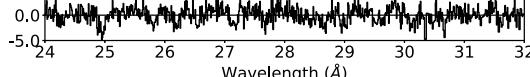
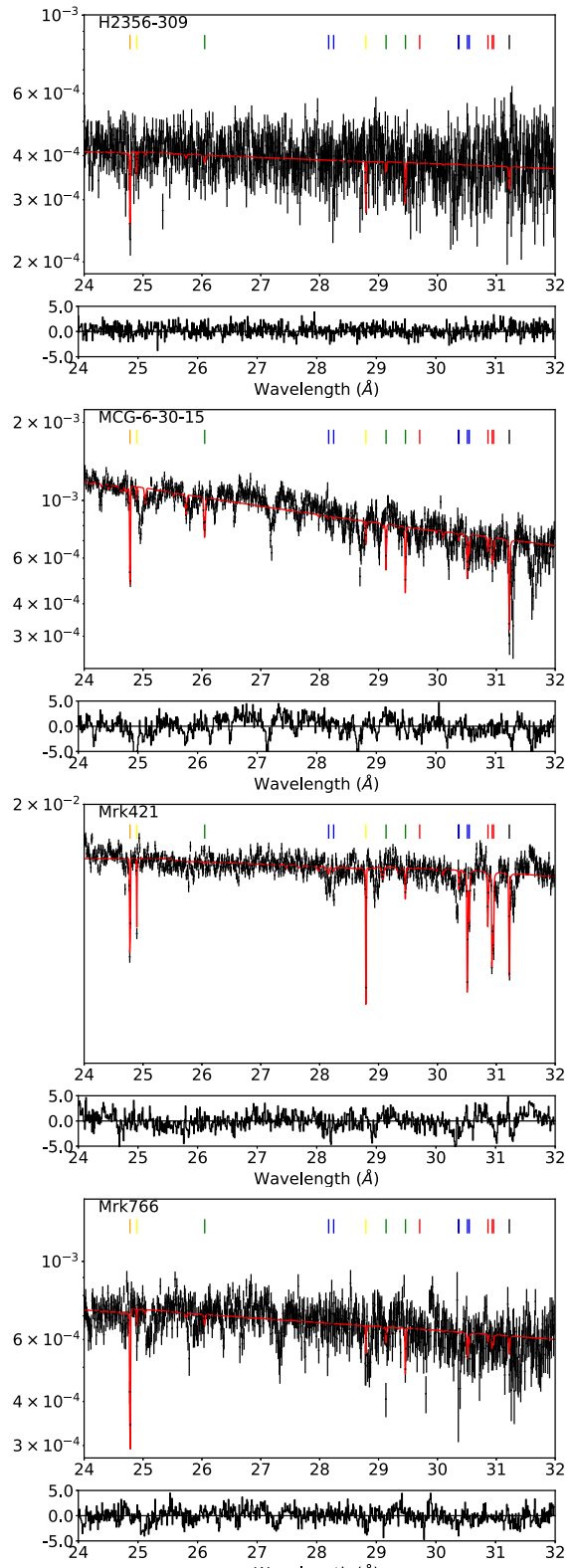

$-5.9 \begin{array}{lllllllll}24 & 25 & 26 & 27 & 28 & 29 & 30 & 31 & 32\end{array}$

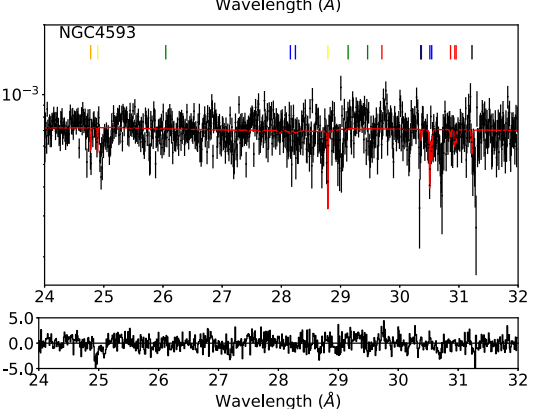

Figure A2. continued 

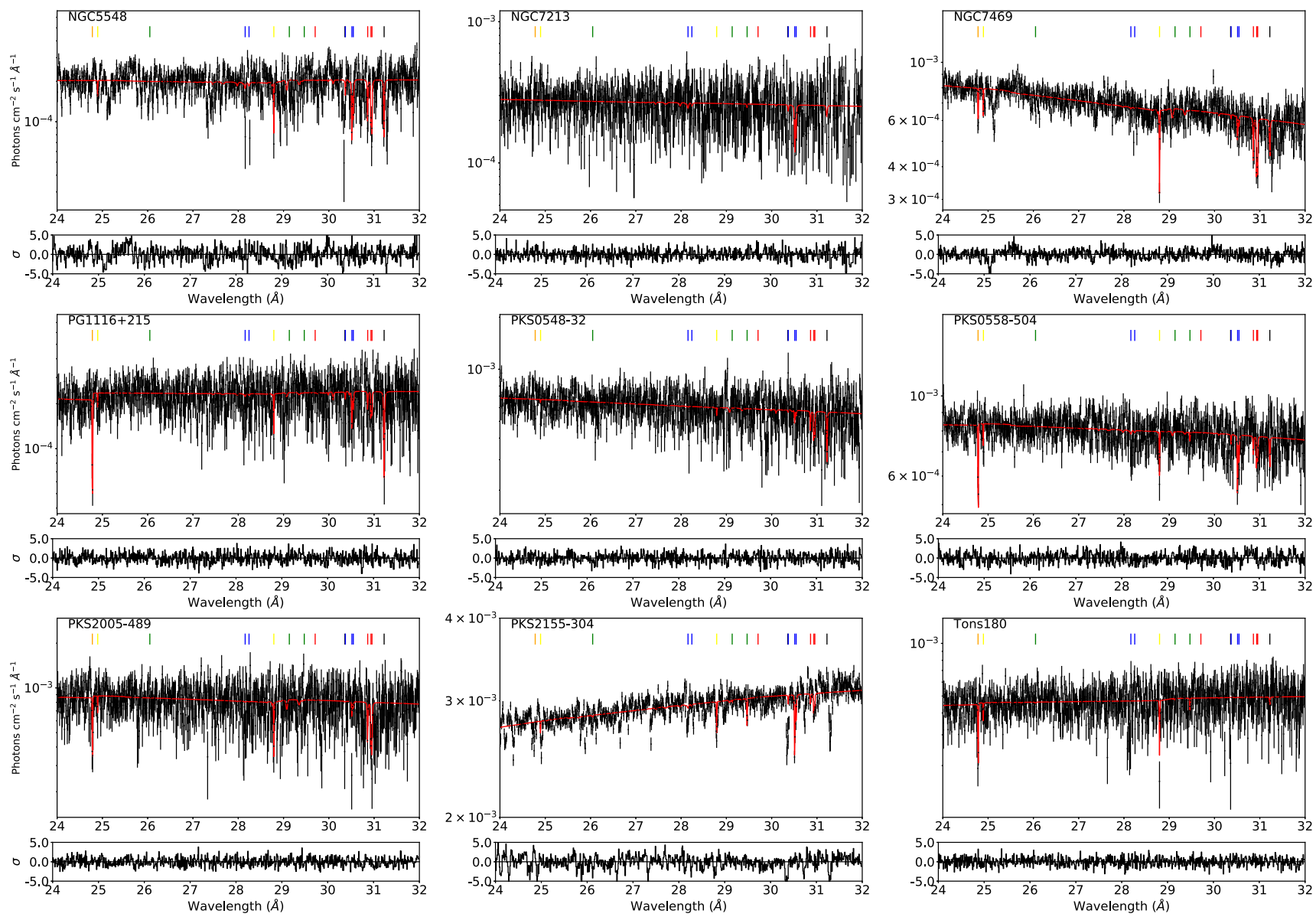

Figure A2. continued 